\title{
Development of a biomarker mortality risk model in acute respiratory distress syndrome
}

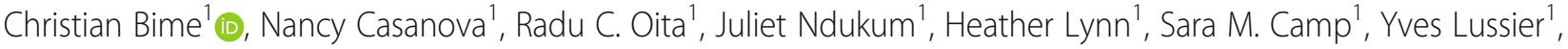 \\ Ivo Abraham², Darrick Carter ${ }^{3}$, Edmund J. Miller ${ }^{4}$, Armand Mekontso-Dessap ${ }^{5}$, Charles A. Downs ${ }^{6}$ and \\ Joe G. N. Garcia ${ }^{1 *}$
}

\begin{abstract}
Background: There is a compelling unmet medical need for biomarker-based models to risk-stratify patients with acute respiratory distress syndrome. Effective stratification would optimize participant selection for clinical trial enrollment by focusing on those most likely to benefit from new interventions. Our objective was to develop a prognostic, biomarker-based model for predicting mortality in adult patients with acute respiratory distress syndrome.

Methods: This is a secondary analysis using a cohort of 252 mechanically ventilated subjects with the diagnosis of acute respiratory distress syndrome. Survival to day 7 with both day 0 (first day of presentation) and day 7 sample availability was required. Blood was collected for biomarker measurements at first presentation to the intensive care unit and on the seventh day. Biomarkers included cytokine-chemokines, dual-functioning cytozymes, and vascular injury markers. Logistic regression, latent class analysis, and classification and regression tree analysis were used to identify the plasma biomarkers most predictive of 28-day ARDS mortality.

Results: From eight biologically relevant biomarker candidates, six demonstrated an enhanced capacity to predict mortality at day 0 . Latent-class analysis identified two biomarker-based phenotypes. Phenotype A exhibited significantly higher plasma levels of angiopoietin-2, macrophage migration inhibitory factor, interleukin-8, interleukin-1 receptor antagonist, interleukin-6, and extracellular nicotinamide phosphoribosyltransferase (eNAMPT) compared to phenotype B. Mortality at 28 days was significantly higher for phenotype A compared to phenotype B (32\% vs 19\%, $p=0.04$ ).
\end{abstract}

Conclusions: An adult biomarker-based risk model reliably identifies ARDS subjects at risk of death within 28 days of hospitalization.

Keywords: ARDS, Predictive analytics, Biomarkers, Mortality

\section{Introduction}

Acute respiratory distress syndrome (ARDS) is a devastatingly-intense inflammatory lung disorder characterized by severe respiratory failure requiring mechanical ventilation with a high mortality rate of $30-40 \%$ [1]. ARDS exhibits clinical and biological heterogeneity with respiratory and multi-organ system failure in response to diverse inciting stimuli. For example, in the USA, sepsis and pneumonia are the major inducers of ARDS, whereas in India, ARDS occurs in response to various

\footnotetext{
* Correspondence: skipgarcia@email.arizona.edu

${ }^{1}$ College of Medicine, University of Arizona Health Sciences, Tucson, AZ, USA Full list of author information is available at the end of the article
}

tropical infections (malaria, miliary tuberculosis, dengue infections, etc.) [2]. The clinical and radiographic diagnostic criteria for ARDS are relatively imprecise [3]. Diagnostic uncertainty in ARDS further exacerbates disease heterogeneity and is a potential source of bias in conducting clinical trials. Consequently, the development of novel therapies in ARDS has been extremely challenging and contributes to the abysmal track record of Phase II/III ARDS clinical trials [4]. Scoring systems in critically ill patients such as the APACHE II score [5] or the lung injury severity score [6] successfully link to patient outcomes but fail to provide consistent and accurate predictive estimates of the risk of death in patient populations with a specific disease process.

C The Author(s). 2019 Open Access This article is distributed under the terms of the Creative Commons Attribution 4.0 International License (http://creativecommons.org/licenses/by/4.0/), which permits unrestricted use, distribution, and 
General severity scores lack specificity by failing to distinguish sepsis, ARDS, or acute kidney injury and are not significantly different between ARDS survivors and nonsurvivors [7]. Furthermore, general severity scores do not have pathophysiologic input and, therefore, are unlikely to guide personalized therapies. Attempts to characterize predictors of death in ARDS by developing a prognostic index [8] remain controversial and have not yet been replicated or validated. Thus, there is a compelling unmet medical need to identify clinical and/or biochemical disease-specific parameters that risk-stratify patients for both accurate prognostication and clinical trial purposes. Stratification of ARDS patients with reliable biomarkers predictive of mortality would optimize participant selection for clinical trial enrollment by focusing on those most likely to benefit from new clinical interventions $[9,10]$.

We and others have used "omic" approaches and translational systems biology methods to identify a panel of plasma protein biomarkers with diverse biological mechanisms that suggest a possible association with ARDS mortality [11-22]. The objective of the current study was to utilize plasma protein biomarkers to derive a prognostic model for predicting ARDS patients least likely to survive to 28 days. We used latent class analysis and predictive analytics on eight plasma biomarkers collected from a wellphenotyped combined ARDS cohort. Biomarkers included cytokine-chemokines (interleukin-6 [IL-6], interleukin-8 [IL-8], interleukin-1 beta [IL-1B], and interleukin-1 receptor antagonist [IL-1RA]), dual-functioning cytozymes, i.e., cytokine/intracellular enzymes (macrophage migration inhibitory factor [MIF], nicotinamide phosphoribosyltransferase [eNAMPT] ) and vascular injury markers (sphingosine 1-phosphate receptor 3 [S1PR3], angiopoietin-2 [Ang-2]). These analyses utilizing complementary analytical approaches, latent class analysis, classification and regression tree (CART) analysis, and rank aggregation were consistent and allowed the identification of six biomarkers that were most predictive of ARDS mortality.

\section{Materials and methods}

\section{Source of data}

Clinical data and plasma samples from 252 well-phenotyped ARDS patients including 203 patients enrolled in the Fluid and Catheter Treatment Trial (FACTT) study [23], and 49 ARDS patients enrolled at the University of Arizona and University of Illinois (IRB\#1312168664R001 and \#20120192 respectively) were studied.

\section{Participants}

All patients with ARDS who met the diagnostic criteria per the American-European Consensus Conference (AECC) [24] or the Berlin definition [3] were included.

\section{Outcome}

The primary outcome was mortality by day 28 .

\section{Predictors}

Initial (D0) plasma samples were taken within $48 \mathrm{~h}$ of meeting ARDS criteria, and for those who survived, a second sample was collected on the seventh day (D7) for biomarker measurements. The plasma levels of the following biomarkers were measured: IL-6, IL-8, IL-1RA, IL-1B, MIF, eNAMPT, S1PR3, and Ang-2.

\section{Blood collection and plasma biomarker measurements}

Blood was collected within $48 \mathrm{~h}$ of ARDS onset (defined as time of meeting all Berlin Criteria) in EDTA-treated tubes, centrifuged within $1 \mathrm{~h}$ from sample collection $(2000 \times g$ for $20 \mathrm{~min}, \mathrm{RCF})$ and stored at $-80^{\circ} \mathrm{C}\left(-70^{\circ} \mathrm{C}\right.$ for FACTT samples). Plasma concentrations of four biomarkers (IL-6, IL-8, IL-1B, IL-1RA) were measured in duplicate using a custom Bio-Plex Pro Human Cytokine 5-plex immunoassay (Bio-Rad, Hercules, CA) and Bio-Plex MAGPIX instrument following the manufacturer's guidelines. Enzyme-linked immunosorbent assay (ELISA) techniques were utilized to quantify plasma levels of eNAMPT (an internally developed ELISA [25]), MIF (R\&D System ${ }^{\circ}$, Minneapolis, MN), S1P3 [26], and Ang-2 (R\&D System ${ }^{\circ}$, Minneapolis, MN) using commercially available ELISAs according to the manufacturer's instructions.

\section{Statistical analysis \\ Exploratory analysis}

Exploratory data analysis was performed for each biomarker (baseline and day 7). Median values and first and third quartiles were calculated for the continuous variables. Percentage values were computed and reported for categorical variables. The signed rank test was performed to assess the differences between initial biomarker measurements compared to those taken 7 days later.

\section{Logistic regression}

To identity biomarkers that potentially significantly contribute to mortality, binary logistic regression and a multiple forward stepwise logistic regression was constructed with APACHE, IL-6, IL-8, IL-1RA, IL-1B, MIF, eNAMPT, S1PR3, and Ang-2 as covariates. In addition, a binary logistic regression was performed, where mortality was fit to the difference in measurements (D0 and D7) for each biomarker to determine whether the mortality status of patients is affected by a change in individual biomarker measurements.

\section{Latent class analysis}

Eight plasma biomarkers (D0) were included as inputs in the latent class analysis (LCA) model. A series of latent class models were fitted with different class sizes, specifically, class 
sizes of two, three, four, and five. The criteria for selecting the optimal number of classes were based on Bayesian Information Criteria (BIC), the Vuong-Lo-Mendell-Rubin (VLMR) likelihood ratio test, and the size of the smallest class. Latent class model estimation was based on fullinformation maximum likelihood methods as implemented in Mplus [9]. To understand how the biomarkers distinguished each class, study participants were assigned to their most likely class and the mean values of the biomarkers compared by class assignment. Once the number of classes was determined, we tested the association between class assignment and 28-day mortality.

\section{Classification and regression trees (CART)}

CART analysis has been used in many areas for decisionmaking purposes to develop models that can classify and predict subjects into various risk categories [27]. We used CART analysis to determine the optimal combination of biomarkers and cut-offs predictive of mortality. The optimal classification tree was derived with a random $80 \%$ sampling of ARDS patients with D0 biomarker measurements, while imposing a condition to the CART algorithm of a minimum of 15 measurements needed to split a node. Terminal nodes that did not improve the classification based on class probability method were pruned. Weighting of cases and costs for misclassification were not used. The remaining $20 \%$ of patients with D0 biomarker measurements were used for testing. As the test data (50 patients) was underpowered to generate a meaningful CART algorithm, we used resampling techniques to create a new data consisting of 200 patients. This was performed by randomly selecting individuals with replacement. CART analysis was performed on the resampled data and test diagnostics calculated. Diagnostic statistics, presented as sensitivity, specificity, positive predictive value, and negative predictive value of the risk classification model, were computed separately for the derivation and test cohorts.

\section{Ranking of biomarkers by importance after CART analysis}

We tested ten iterations of the data in order to rank the biomarkers by importance to the classification trees: (1) all D0 and D7 biomarker measurements combined, (2) D0 biomarker measurements only, (3) first half of D0 biomarker measurements, (4) second half of D0 biomarker measurements, (5) first half of D7 biomarker measurements, (6) second half of D7 measurements, (7) D7 measurements only, (8) $80 \%$ random sample of D0 cohort. (Additional file 2: Table S1). For each iteration, the following conditions were imposed: (i) 15 or greater observations required for a nodal split and (ii) splits must decrease the overall lack of fit by a factor of $1 \mathrm{e}-5$ (or $0.001 \%$ ). The analyzed results from each of the 20 created CART outputs includes a list of biomarkers arranged by the importance to the classification tree along with an associated numeric score where an elevated score indicates increasing biomarker importance in predicting mortality. Finally, a rank aggregation algorithm [28] was used to generate final ranking of biomarkers by importance for each ordered list. All analyses were performed in R (R Core Team, 2018) and Mplus v8.3 [29].

\section{Results}

\section{Characteristics of the cohort}

The demographic and clinical characteristics of the ARDS subjects are presented in Table 1. Mortality among ARDS patients was $22 \%$ with non-survivors significantly older than survivors. There were no other significant differences in the clinical characteristics between ARDS survivors and non-survivors (Table 1). The medians (IQR) for all eight biomarkers for ARDS patients at day 0 and day 7 are presented in Table 2. Of the eight biomarkers, only eNAMPT was significantly higher on day 7 compared to day 0 (35\% higher) (Table 2). Values of IL-6, IL-1RA, IL-8, Ang-2, and S1PR3 were significantly higher on day 0 compared to day 7. There was no significant difference between levels of MIF and IL-1B on days 0 and 7.

\section{Results of logistic regression}

Logistic regression analysis assessing the ability of each biomarker measured at D0 to predict mortality failed to show any biomarker to be statistically significant after adjustment for covariates (Additional file 2: Table S2). For measurements taken at D7, only Ang-2 levels were significantly associated with an increase in mortality

Table 1 Demographics and clinical characteristics of the ARDS cohort

\begin{tabular}{lllc}
\hline Variable & \multicolumn{2}{l}{ ARDS cohort } & P value \\
\cline { 2 - 3 } & Alive $=197$ & Dead $=55$ & \\
\hline Sex, female, N (\%) & $100(51 \%)$ & $22(40 \%)$ & 0.16 \\
Age, median (Q1, Q3) & $48(39,56)$ & $58(49,70)$ & $<0.0001$ \\
APACHE II Score, median (Q1, Q3) & $76(56,101)$ & $91(28,124)$ & 0.10 \\
Race/ethnicity, N (\%) & & & \\
Black or African American & $30(15 \%)$ & $16(29 \%)$ & 0.05 \\
White & $163(83 \%)$ & $39(71 \%)$ & \\
Other* & $4(2.0 \%)$ & $0(0 \%)$ & \\
ARDS etiology, N (\%) & & & 0.14 \\
Sepsis & $89(45 \%)$ & $31(56 \%)$ & 1.0 \\
Trauma & $17(8.6 \%)$ & $5(9 \%)$ & 0.7 \\
Pneumonia & $127(64 \%)$ & $37(67 \%)$ & \\
Source of cohort data & & & \\
FACTT cohort & 162 & 41 & \\
University of Illinois cohort & 8 & 9 & \\
University of Arizona cohort & 27 & 5 & \\
\hline
\end{tabular}

*Other indicates: any race/ethnicity other than Black or White FACTT Fluids and Catheters Treatment Trial 
Table 2 Biomarker plasma levels at day 0 and at day 7 in ARDS cohort

\begin{tabular}{llll}
\hline Biomarker & D0 median $(\mathrm{Q} 1, \mathrm{Q} 3)(\mathrm{N})$ & D7 median $(\mathrm{Q} 1, \mathrm{Q})(\mathrm{N})$ & $P$ value \\
\hline IL-6 $(\mathrm{pg} / \mathrm{ml})$ & $27(7,92) 145$ & $12(4,54) 115$ & $<0.01$ \\
IL-8 $(\mathrm{pg} / \mathrm{ml})$ & $150(67,359) 201$ & $108(44,298) 200$ & 0.001 \\
IL-1RA $(\mathrm{pg} / \mathrm{ml})$ & $3072(1241,6545) 229$ & $2755(1067,5695) 225$ & 0.04 \\
MIF $(\mathrm{ng} / \mathrm{ml})$ & $45(29,78) 242$ & $49(31,84) 247$ & 0.07 \\
NAMPT $(\mathrm{ng} / \mathrm{ml})$ & $60(34,74) 248$ & $81(55,113) 248$ & $<0.001$ \\
S1PR3 $(\mathrm{ng} / \mathrm{ml})$ & $577(227,1458) 231$ & $399(97,1066) 233$ & $<0.001$ \\
Ang-2 $(\mathrm{ng} / \mathrm{ml})$ & $12(7,23) 247$ & $7(4,12) 247$ & $<0.001$ \\
IL-1B $(\mathrm{pg} / \mathrm{ml})$ & $44(24,68) 228$ & $39(20,61) 233$ & $<0.01$ \\
\hline
\end{tabular}

${ }^{1} P$ value from Wilcoxon signed rank test comparing the measurements for D0 and D7 for all eight biomarkers. $I L-6$ interleukin- $6, I L-8$ interleukin-8, IL-1RA interleukin-1 receptor antagonist, MIF Macrophage migration inhibitory factor, NAMPT nicotinamide phosphoribosyltransferase, S1PR3 sphingosine 1-phosphate receptor 3, Ang-2 angiopoietin-2, IL-1B interleukin 1 beta

(Additional file 2: Table S3). There was no significant association between the change in biomarker measurements taken at the two time points and mortality (Additional file 2: Table S4).

\section{Latent-class modeling and characteristics of each phenotype}

A summary of the model fits for two through five classes is presented in Additional file 2: Table S5 with a two class model for the eight biomarkers providing the optimal fit. The average latent class probabilities were 0.77 for phenotype $\mathrm{B}$ and 0.23 for phenotype A (Additional file 1: Figure S1). Compared to phenotype B, phenotype A exhibited considerably higher plasma levels of Ang-2, IL-6, eNAMPT, MIF, IL-8, and IL-1RA (Fig. 1). There was no significant phenotype difference between levels of IL-1B and S1PR3. In order to determine whether the two phenotypes exhibit differing natural histories, we tested the association between each phenotype and 28-day mortality. Phenotype A subjects exhibited significantly higher mortality compared to phenotype B subjects $(32 \%$ vs $19 \%, p=0.04)$.

\section{Derivation and testing of classification tree}

Maximum accuracy for predicting ARDS mortality was achieved with four of the eight candidate biomarkers: MIF, IL-8, IL-6, and eNAMPT (Fig. 2). No single demographic or clinical variable improved ARDS mortality prediction accuracy. Two low risk nodes (TN1 and TN3, mortality $15 \%$ and $17 \%$ respectively), two medium risk nodes (TN2 and TN4, mortality $24 \%$ and $45 \%$, respectively), and one high risk node (TN5 with mortality of 83\%) were identified. The test characteristics of the biomarker-based ARDS Mortality Risk Stratification Model decision trees are shown in Table 3.

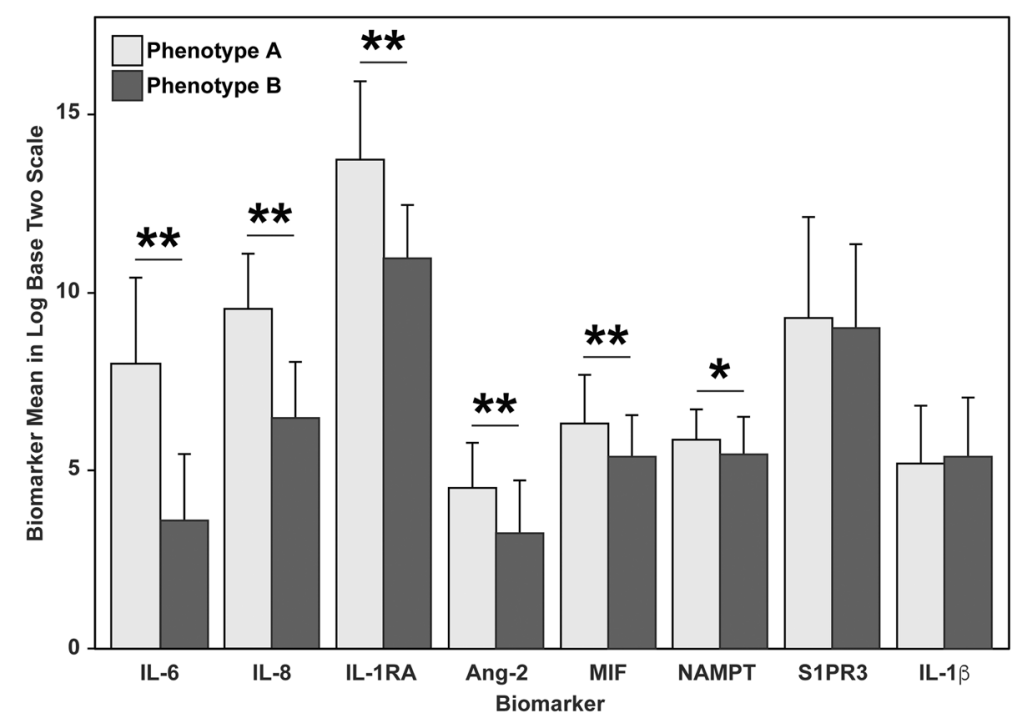

Fig. 1 Differences in biomarker levels by phenotype. Graph showing estimated means for eight biomarkers. Phenotype A is characterized by higher levels of Ang-2, MIF, IL-8, IL-1RA, IL-6, and NAMPT compared to phenotype B 


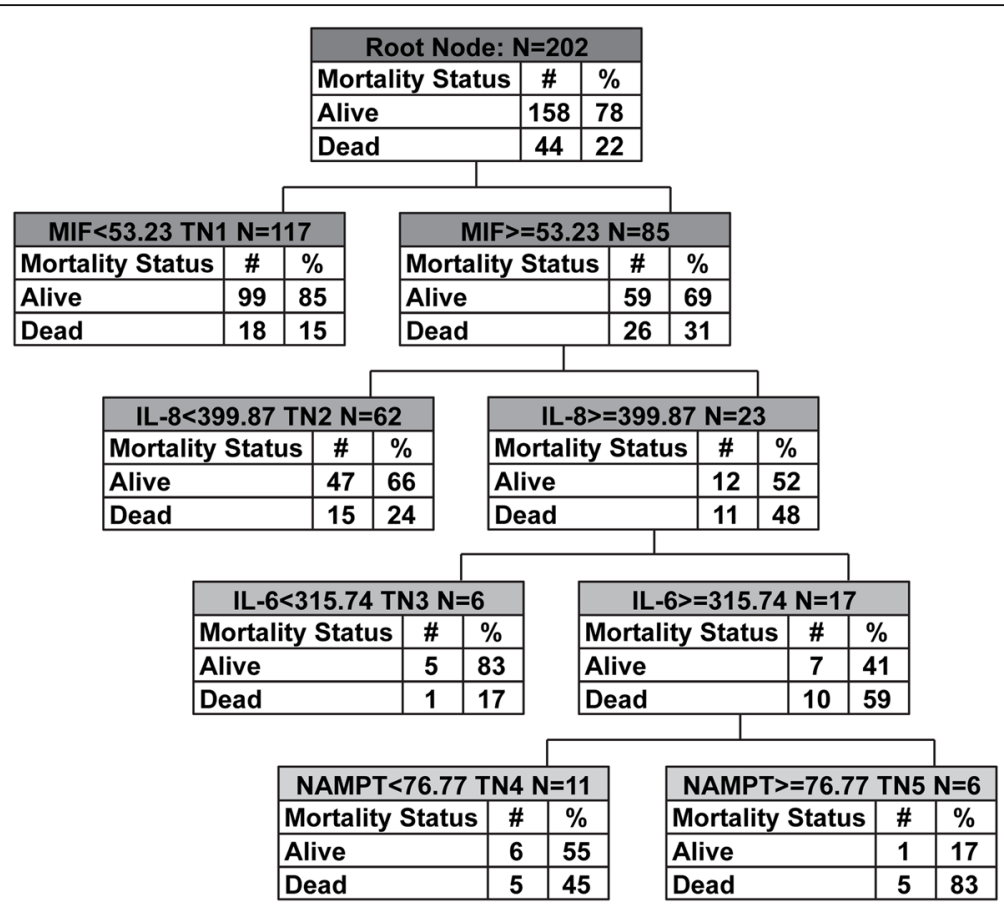

Fig. 2 Biomarker-based ARDS Mortality Risk Stratification Model decision tree. The derived biomarker-based ARDS Mortality Risk Stratification Model decision tree from the random $80 \%$ derivation cohort ( $n=202,44$ non-survivors). The tree contains the mortality probability, macrophage migration inhibitory factor (MIF), interleukin 8 (IL-8), interleukin 6 (IL-6), and extracellular nicotinamide phosphoribosyltransferase (NAMPT). Biomarker concentrations are expressed in ng/ml. Terminal nodes (TN) 1 and TN3 were low risk (15\% and 17\% risk of death), TN2 and TN4 were intermediate risk (24\% and $45 \%$ risk of death, respectively), and TN5 high risk (85\% risk)

\section{Ranking of biomarkers by importance}

Output from CART analysis allowed for a ranking of biomarkers by order of importance in terms of predicting mortality resulting in identification of the top six biomarkers: Ang-2, MIF, IL-8, IL-1RA, IL-6, and eNAMPT (Additional file 2: Table S6).

\section{Discussion}

We initially included eight ARDS-relevant plasma biomarkers derived from specific pathobiologic groups including: (i) inflammatory cytokine-chemokines (IL-6, IL-8, IL-1B, and IL-1RA), (ii) dual-functioning cytozymes, i.e., an intracellular enzyme that also functions as a cytokine when secreted (macrophage migration inhibitory factor, eNAMPT), and iii) vascular injury markers (S1PR3, Ang2). Our findings indicate that a panel of six biomarkers potentially predict ARDS survival. In the latent class modeling we performed, a biomarker-based phenotype with higher plasma levels of Ang-2, MIF, IL-8, IL-1RA, IL-6, and eNAMPT was associated with significantly higher mortality (Fig. 1). The complementary CART analysis revealed that the maximum accuracy for predicting high mortality was achieved by four out of these six biomarkers (MIF, IL-8, IL-6, and eNAMPT) (Fig. 2). Finally, rank

Table 3 Test characteristics of the biomarker-based ARDS Mortality Risk Stratification Model decision trees

\begin{tabular}{|c|c|c|c|c|}
\hline \multirow[t]{2}{*}{ Variables } & \multicolumn{2}{|c|}{ Derivation, $80 \%$ day $0,(n=202,44$ non-survivors) } & \multicolumn{2}{|c|}{ Test, $20 \%$ day 0 , resampled to $n=200$} \\
\hline & Values & $95 \% \mathrm{Cl}$ & Values & $95 \% \mathrm{Cl}$ \\
\hline True negatives & 5 & - & 28 & - \\
\hline False positives & 19 & - & 7 & - \\
\hline True positives & 104 & - & 131 & - \\
\hline False negatives & 1 & - & 8 & - \\
\hline Sensitivity & 0.99 & $(0.95,0.99)$ & 0.94 & $(0.88,0.97)$ \\
\hline Specificity & 0.21 & $(0.07,0.42)$ & 0.80 & $(0.63,0.97)$ \\
\hline Positive predictive value & 0.85 & $(0.81,0.87)$ & 0.95 & $(0.91,0.97)$ \\
\hline Negative predictive value & 0.83 & $(0.39,0.98)$ & 0.78 & $(0.64,0.87)$ \\
\hline
\end{tabular}


aggregation identified Ang-2, MIF, IL-8, IL-1RA, IL-6, and eNAMPT as the most important in contributing to mortality (Additional file 2: Table S6).

The pathogenesis of ARDS includes a combination of endothelial injury, epithelial injury, an intense inflammatory cascade, dysregulated coagulation, fibrosis, and apoptosis in response to diverse stimuli. Studies exploring the byproducts of acute dysregulation of various cellular pathways have generated more than 45 potential biomarkers [30, 31]. However, no single biomarker or clinical variable has demonstrated adequate prognostic or predictive ability to identify subphenotypes of ARDS [9, 10, 32, 33]. The ability to stratify ARDS patients by pathobiology and likelihood of treatment response would greatly enrich future clinical trials and enhance the ability to detect a treatment effect [34]. Subphenotyping/endotyping has been successfully accomplished in airways diseases such as asthma and COPD with important therapeutic implications $[35,36]$ and may exist within severe sepsis $[4,37,38]$. However, there is a paucity of data elucidating ARDS sub-phenotypes/endotypes. Recent studies $[9,39]$, utilizing two ARDSnet cohorts, identified two ARDS sub-phenotypes that markedly differed in natural history, clinical and biological characteristics, biomarker profiles, response to positive end-expiratory pressure (PEEP), and ventilator- and organ failure free days and in mortality. The hyperinflammatory ARDS sub-phenotype is characterized by a higher prevalence of sepsis and severe shock, high plasma levels of inflammatory biomarkers (IL-6, IL-8, etc.), greater vasopressor use, and metabolic acidosis. In contrast, the low inflammatory ARDS sub-phenotype exhibited less severe inflammation and shock. Surprisingly, the level of ARDS severity ( $\mathrm{PaO} 2 / \mathrm{FiO} 2$ ratio), renal or hepatic injury severity, or leukocytosis level failed to distinguish these two phenotypes [9]. The hyperinflammatory phenotype was associated with higher mortality, fewer ventilator-free and organ failure-free days, and altered responses to ventilator strategies when compared to the low inflammatory phenotype [40]. Importantly, no single clinical or biological variable was sufficient to identify the sub-phenotype including the severity of ARDS APACHE scores ( $\mathrm{PaO} 2 / \mathrm{FiO} 2$ ratio), severity of renal or hepatic failure, or leukocytosis, suggesting that phenotype membership was not merely a reflection of severity of illness as measured by traditional indices [40].

Our findings are strengthened by the use of three complementary approaches, including predictive analytics, to identify a common biomarker combination that is important in predicting ARDS survival. We also used a well-phenotyped cohort of ARDS patients, the majority of whom were enrolled as part of a large multicenter trial. Therefore, the participants represent a demographically diverse cohort of patients with ARDS strengthening the generalizability of our findings.

Our study has several limitations including the relative low observed mortality of $22 \%$ in the examined cohort compared to the expected ARDS mortality of $30-40 \%$. This is likely due to the selection criteria we utilized where only patients surviving to day 7 were included in order to analyze both days 0 and 7 samples, i.e., patients who died before day 7 were not included. A second limitation of our work is that we had limited statistical power to detect significant differences in the predictive modeling algorithms. Finally, we recognize the lack of an external validation of our ARDS biomarker-based risk model in an independent cohort. Clearly, despite our utilization of a random sample of $80 \%$ of ARDS patients at D0 for the derivation and resampling technique on the remaining $20 \%$ of patients to demonstrate good test characteristics (Table 3), we plan to next evaluate our mortality panel an independent cohort to determine the reproducibility of these six biomarkers in predicting ARDS survival.

\section{Conclusion}

We have used complementary analytic approaches to identify six biomarkers (Ang-2, MIF, IL-8, IL-1RA, IL-6, eNAMPT) that show promise in predicting survival in ARDS. These biomarkers provide an important proof-ofconcept that a combination of ARDS biomarkers can improve sub-phenotyping and enhance predictive and prognostic ability at the time of diagnosis. This information could be useful for guiding enrollment in clinical trials and represents an important step towards better patient stratification at the time of presentation to enhance the likelihood of a positive clinical trial in this vexing disorder.

\section{Trial status}

Not applicable.

\section{Supplementary information}

Supplementary information accompanies this paper at https://doi.org/10. 1186/s13054-019-2697-x

Additional file 1: Figure S1. Probability of Latent Class Assignment. Barplot of probability of class assignment using eight biomarkers.

Additional file 2: Table S1. Ranking algorithm. Eight CART iterations, algorithm conditions imposed, and number of biomarkers used. Table S2. Logistic regression analysis of mortality prediction capacity of the eight ARDS biomarkers-Day Zero. Table S3. Logistic regression analysis of mortality prediction capacity of the eight ARDS biomarkers-Day Seven. Table S4. Logistic regression analysis to assess the mortality prediction for change in measurements for the eight biomarkers. Table S5. Number of individuals per latent class. Table S6. Ranking of ARDS biomarker importance in predicting mortality.

\section{Acknowledgements}

We acknowledge the investigators of the ARDSnet FACTT study for the data used in this study.

Authors' contributions

$C B, N C, R C O, I A, Y L, D C$, and JGNG contributed to the conception and design of the work, the analysis and interpretation of data for the work, the drafting and revision of the manuscript, and approval of final version to be published. 
EJM and CAD contributed to the conception and design of the work, revision of the manuscript, and approval of final version to be published. JN contributed to the conception and design of the work, the analysis and interpretation of data for the work, critical revision of key intellectual content, and approval of final version to be published. HL and SC collected data, contributed to the analysis and interpretation of data for the work, and assisted with processing and manuscript revision. All authors read and approved the final manuscript.

\section{Funding}

This work was supported by K08 HL141623 (CB); P01 HL126609 (JGNG), R41 HL147769 (JGNG); and R42 HL145930 (JGNG).

\section{Availability of data and materials}

Yes. Primary source data and methods are available.

\section{Ethics approval and consent to participate}

We had the following ethical approvals: for patients enrolled at the University of Arizona (IRB\#1312168664R001) and at the University of Illinois IRB\#20120192. Informed consent was obtained from each participant or their surrogate.

The ARDSnet FACTT study had ethical approval and consent from all participants in each center

\section{Consent for publication}

Yes. We the authors give consent for publication.

\section{Competing interests}

The authors declare that they have no competing interests.

\section{Author details}

'College of Medicine, University of Arizona Health Sciences, Tucson, AZ, USA. ${ }^{2}$ College of Pharmacy, University of Arizona Health Sciences, Tucson, USA. ${ }^{3}$ PAI Life Sciences, Seattle, WA, USA. ${ }^{4}$ RDS2 Solutions, Stony Brook, NY, USA. ${ }^{5}$ Medical Intensive Care Unit, Henri Mondor Hospital, AP-HP, 94010 Créteil, France. ${ }^{6}$ College of Nursing and Health Sciences, University of Florida, Gainesville, USA.

Received: 5 September 2019 Accepted: 9 December 2019 Published online: 16 December 2019

\section{References}

1. Bellani G, Laffey JG, Pham T, et al. Epidemiology, patterns of care, and mortality for patients with acute respiratory distress syndrome in intensive care units in 50 CountriesTrends in acute respiratory distress syndrome in 50 CountriesTrends in acute respiratory distress syndrome in 50 countries. JAMA. 2016;315(8):788-800

2. Swaroopa D, Bhaskar $K$, Mahathi T, et al. Association of serum interleukin-6, interleukin-8, and acute physiology and chronic health evaluation II score with clinical outcome in patients with acute respiratory distress syndrome. Indian J Crit Care Med. 2016:20(9):518-25.

3. Ranieri VM, Rubenfeld GD, Thompson BT, et al. Acute respiratory distress syndrome: the Berlin definition. JAMA. 2012;307(23):2526-33.

4. Frank AJ, Thompson BT. Pharmacological treatments for acute respiratory distress syndrome. Curr Opin Crit Care. 2010;16(1):62-8.

5. Knaus WA, Draper EA, Wagner DP, Zimmerman JE. APACHE II: a severity of disease classification system. Crit Care Med. 1985:13(10):818-29.

6. Murray JF, Matthay MA, Luce JM, Flick MR. An expanded definition of the adult respiratory distress syndrome. Am Rev Respir Dis. 1988;138(3):720-3.

7. Kangelaris KN, Calfee CS, May AK, Zhuo H, Matthay MA, Ware LB. Is there still a role for the lung injury score in the era of the Berlin definition ARDS? Ann Intensive Care. 2014:4(1):4

8. Ware LB. Prognostic determinants of acute respiratory distress syndrome in adults: impact on clinical trial design. Crit Care Med. 2005; 33(3 Suppl):S217-22.

9. Calfee CS, Delucchi K, Parsons PE, Thompson BT, Ware LB, Matthay MA. Subphenotypes in acute respiratory distress syndrome: latent class analysis of data from two randomised controlled trials. Lancet Respir Med. 2014;2(8):611-20.
10. Calfee $\mathrm{CS}$, Delucchi $\mathrm{KL}$, Sinha $\mathrm{P}$, et al. Acute respiratory distress syndrome subphenotypes and differential response to simvastatin: secondary analysis of a randomised controlled trial. Lancet Respir Med. 2018;6(9):691-8.

11. Goldman JL, Sammani S, Kempf C, et al. Pleiotropic effects of interleukin-6 in a "two-hit" murine model of acute respiratory distress syndrome. Pulm Circ. 2014:4(2):280-8.

12. Kurdowska AK, Geiser TK, Alden SM, et al. Activity of pulmonary edema fluid interleukin-8 bound to alpha(2)-macroglobulin in patients with acute lung injury. Am J Physiol Lung Cell Mol Physiol. 2002;282(5):L1092-8.

13. Krupa A, Kato H, Matthay MA, Kurdowska AK. Proinflammatory activity of anti-IL-8 autoantibody: IL-8 complexes in alveolar edema fluid from patients with acute lung injury. Am J Physiol Lung cell Mol Physiol. 2004;286(6): L1105-13.

14. Dahmer MK, Quasney MW, Sapru A, et al. Interleukin-1 receptor antagonist is associated with pediatric acute respiratory distress syndrome and worse outcomes in children with acute respiratory failure. Pediatr Crit Care Med. 2018:19(10):930-8.

15. Meduri GU, Headley S, Kohler G, et al. Persistent elevation of inflammatory cytokines predicts a poor outcome in ARDS. Plasma IL-1 beta and IL-6 levels are consistent and efficient predictors of outcome over time. Chest. 1995; 107(4):1062-73.

16. Lai KN, Leung JC, Metz CN, Lai FM, Bucala R, Lan HY. Role for macrophage migration inhibitory factor in acute respiratory distress syndrome. J Pathol. 2003:199(4):496-508

17. Hernandez-Pacheco N, Guillen-Guio B, Acosta-Herrera M, et al. A vascular endothelial growth factor receptor gene variant is associated with susceptibility to acute respiratory distress syndrome. Intensive Care Med Exp. 2018;6(1):16

18. Jabaudon M, Berthelin P, Pranal T, et al. Receptor for advanced glycation end-products and ARDS prediction: a multicentre observational study. Sci Rep. 2018;8(1):2603.

19. Lutz W, Stetkiewicz J. High mobility group box 1 protein as a late-acting mediator of acute lung inflammation. Int J Occup Med Environ Health. 2004:17(2):245-54

20. Ye SQ, Simon BA, Maloney JP, et al. Pre-B-cell colony-enhancing factor as a potential novel biomarker in acute lung injury. Am J Respir Crit Care Med. 2005;171(4):361-70.

21. Sun X, Ma SF, Wade MS, et al. Functional promoter variants in sphingosine 1-phosphate receptor 3 associate with susceptibility to sepsis-associated acute respiratory distress syndrome. Am J Physiol Lung Cell Mol Physiol. 2013;305(7):L467-77.

22. Zinter MS, Spicer A, Orwoll BO, et al. Plasma angiopoietin-2 outperforms other markers of endothelial injury in prognosticating pediatric ARDS mortality. Am J Phys Lung Cell Mol Phys. 2016;310(3):L224-31.

23. Wiedemann HP, Wheeler AP, Bernard GR, et al. Comparison of two fluidmanagement strategies in acute lung injury. N Engl J Med. 2006;354(24): 2564-75.

24. Bernard G, Artigas A, Brigham K, et al. The American-European Consensus Conference on ARDS: definitions, mechanisms, relevant outcomes, and clinical trial coordination. Am J Respir Crit Care Med. 1994;149:818-24.

25. Oita RC, Camp SM, Ma W et al. Novel mechanism for nicotinamide phosphoribosyltransferase inhibition of TNF-a-mediated apoptosis in human lung endothelial cells. Am J Respir Cell Mol Biol. 2018:59(1):36-44.

26. Sun $X$, Singleton PA, Letsiou $E$, et al. Sphingosine-1-phosphate receptor3 is a novel biomarker in acute lung injury. Am J Respir Cell Mol Biol. 2012;47(5):628-36

27. Krzywinski M, Altman N. Classification and regression trees. Nat Methods. 2017;14:757

28. Pihur V, Datta S, Datta S. RankAggreg, an R package for weighted rank aggregation. BMC Bioinformatics. 2009;10(1):62.

29. Muthen LaMB. Mplus User's Guide, 8. Muthen \& Muthen: Los Angeles. 1998-2017.

30. Blondonnet R, Constantin JM, Sapin V, Jabaudon M. A pathophysiologic approach to biomarkers in acute respiratory distress syndrome. Dis Markers. 2016;2016:3501373.

31. Spadaro S, Park M, Turrini C, et al. Biomarkers for acute respiratory distress syndrome and prospects for personalised medicine. J Inflamm. 2019;16(1):1.

32. Amur S, LaVange L, Zineh I, Buckman-Garner S, Woodcock J. Biomarker qualification: toward a multiple stakeholder framework for biomarker development, regulatory acceptance, and utilization. Clin Pharmacol Ther. 2015;98(1):34-46. 
33. Mayeux R. Biomarkers: potential uses and limitations. NeuroRx. 2004; 1(2):182-8.

34. Barker AD, Compton CC, Poste G. The National Biomarker Development Alliance accelerating the translation of biomarkers to the clinic. Biomark Med. 2014;8(6):873-6.

35. Schivo M, Albertson TE, Haczku A, et al. Paradigms in chronic obstructive pulmonary disease: phenotypes, immunobiology, and therapy with a focus on vascular disease. J Invest Med. 2017;65(6):953-63.

36. Wenzel SE. Asthma phenotypes: the evolution from clinical to molecular approaches. Nat Med. 2012;18(5):716-25.

37. Iskander KN, Osuchowski MF, Stearns-Kurosawa DJ, et al. Sepsis: multiple abnormalities, heterogeneous responses, and evolving understanding. Physiol Rev. 2013;93(3):1247-88

38. Wong HR, Cvijanovich NZ, Allen $\mathrm{GL}$, et al. Validation of a gene expressionbased subclassification strategy for pediatric septic shock. Crit Care Med. 2011;39(11):2511-7.

39. Famous $K R$, Delucchi $K$, Ware $L B$, et al. Acute respiratory distress syndrome subphenotypes respond differently to randomized fluid management strategy. Am J Respir Crit Care Med. 2017;195(3):331-8.

40. Calfee $C$, Matthay M, Eisner M, et al. Active and passive cigarette smoking and acute lung injury after severe blunt trauma. Am J Respir Crit Care Med. 2011:183:1660-5.

\section{Publisher's Note}

Springer Nature remains neutral with regard to jurisdictional claims in published maps and institutional affiliations.

Ready to submit your research? Choose BMC and benefit from:

- fast, convenient online submission

- thorough peer review by experienced researchers in your field

- rapid publication on acceptance

- support for research data, including large and complex data types

- gold Open Access which fosters wider collaboration and increased citations

- maximum visibility for your research: over $100 \mathrm{M}$ website views per year

At $\mathrm{BMC}$, research is always in progress.

Learn more biomedcentral.com/submissions 